\section{Commentary: The rise of minimalism in cardiac surgery}

\author{
Ettorino Di Tommaso, MD, and \\ Vito Domenico Bruno, MD, PhD
}

In the recent years, cardiac surgery and minimal incisions have become an inseparable binomial with the development of new and innovative techniques to minimize the surgical trauma of heart surgery. The number of operations performed with a minimally invasive approach is increasing, with many centers worldwide providing this approach as the standard for a wide range of procedures. ${ }^{1}$ The evolution of minimally invasive surgery has been fast, beginning with initial attempts at coronary artery bypass surgery through limited access and extending to complex and combined procedures. In the current issue of JTCVS Techniques, Shah and colleagues ${ }^{2}$ present their series of aortic root replacements (Bentall) performed through upper mini-sternotomy (UMS), comparing it with the standard full sternotomy Bentall procedure. The authors report a single-center, single-surgeon experience of 48 consecutive mini-Bentall procedures completed over a 10 -year period. The benefits of the Bentall procedure have been largely demonstrated, ${ }^{3}$ but the effectiveness and feasibility of minimally invasive in this type of procedure remain under debate.

The retrospective analysis conducted by Shah and colleagues represent an interesting and remarkable addition to the discussion. Their results show similar outcomes with this approach compared with a selected group of full sternotomy patients who underwent surgery with the same surgeon, at the same institution, over a 10-year period. No

\footnotetext{
From the Translational Health Science, Bristol Medical School, University of Bristol, Bristol, United Kingdom.

Disclosures: The authors reported no conflicts of interest.

The Journal policy requires editors and reviewers to disclose conflicts of interest and to decline handling or reviewing manuscripts for which they may have a conflict of interest. The editors and reviewers of this article have no conflicts of interest.

Received for publication Feb 3, 2021; revisions received Feb 3, 2021; accepted for publication Feb 4, 2021; available ahead of print Feb 8, 2021.

Address for reprints: Vito Domenico Bruno, MD, PhD, Translational Health Sciences, Bristol Medical School, University of Bristol, Research Floor Level 7, Bristol Royal Infirmary, Upper Maudlin St, BS2 8HW Bristol, United Kingdom (E-mail: Vito.D.Bruno@bristol.ac.uk).

JTCVS Techniques 2021;7:67-8

2666-2507

Copyright (C) 2021 The Authors. Published by Elsevier Inc. on behalf of The American Association for Thoracic Surgery. This is an open access article under the CC BY-NCND license (http://creativecommons.org/licenses/by-nc-nd/4.0/).

https://doi.org/10.1016/j.xjtc.2021.02.012
}

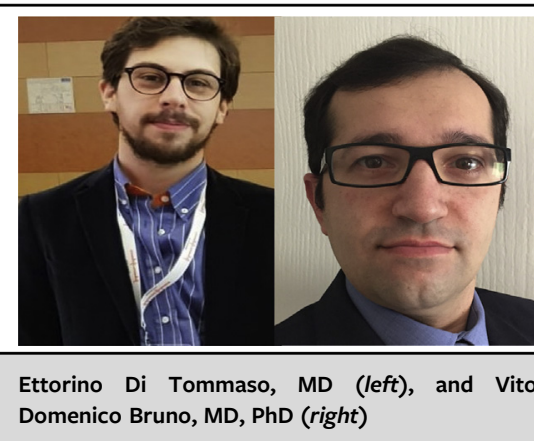

CENTRAL MESSAGE

A minimal surgical approach during a Bentall procedure is an interesting and valid alternative to the classic median sternotomy-a "minimalist" way to perform a major procedure.

significant differences between the 2 groups were observed in terms of cardiopulmonary bypass time, aortic crossclamp time, and intensive care unit length of stay, whereas the mini-Bentall group showed significantly shorter ventilation time and smaller reoperation rates compared which the full Bentall approach and a slightly shorter hospital length of stay. These results confirm the literature data ${ }^{4}$ showing that this technique is safe for aortic root surgery when performed by surgeons with expertise in minimally invasive surgery.

In that regard, one of the article's greatest contributions is the detailed and meticulous description of the surgical technique. Considering the complexity of these procedures, this information is undoubtedly valuable for the surgical community. The authors are keen to underline this factor, highlighting the importance of the step-by-step approach and the importance of gaining enough experience with the upper mini-sternotomy in simpler operations before advancing to more complex aortic surgeries. It must noted that the authors describe a single surgeon experience, which is one of the main limitations. As in many minimal invasive surgeries, even this procedure requires a long, steep learning curve. A dedicated learning pathway for the mini-Bentall would be the way forward, as junior surgeons need a specific training to achieve similar results, and the importance of a dedicated minimally invasive fellowship in a highvolume center has been previously highlighted even for other types of surgeries. ${ }^{1}$ Another limitation of this article 
is represented by the different eras of the 2 groups; whereas the full-Bentall group was represented by operations conducted in a previous surgical era, the mini-Bentall group is more recent, and its potential benefits may be related in part to the advances in clinical and anesthesiologic care achieved over the last few years. Despite this, there is no doubt about the safety and effectiveness of this procedure.

Not surprisingly, we are seeing an ever-increasing interest in "minimalism" in every aspect of cardiac surgery, from minimal invasive coronary artery bypass grafting to mini-sternotomy for aortic valve replacement and from mini-thoracotomy mitral valve surgery to the Bentall operation. Intuitively, a smaller surgical incision should provide immediate benefits for the patient, but stronger evidence and dedicated learning pathways are required to sustain the future of these procedures. In conclusion, it looks like "minimalism" in cardiac surgery is here to stay: not everyone might like it, but many will fall in love with it.

\section{References}

1. Nissen AP, Nguyen S, Abreu J, Nguyen TC. The first 5 years: building a minimally invasive valve program. J Thorac Cardiovasc Surg. 2019;157:1958-65.

2. Shah VN, Kilcoyne MF, Buckley M, Sicouri S, Plestis KA. The mini-Bentall approach: comparison with full sternotomy. J Thorac Cardiovasc Surg Tech. 2021;7:59-66.

3. Etz CD, Bischoff MS, Bodian C, Roder F, Brenner R, Griepp RB, et al. The Bentall procedure: is it the gold standard? A series of 597 consecutive cases. J Thorac Cardiovasc Surg. 2010;140(6 Suppl):S64-70.

4. Mikus E, Micari A, Calvi S, Salomone M, Panzavolta M, Paris M, et al. Mini-Bentall an interesting approach for selected patients. Innovations (Phila). 2017;12: $41-5$. 